\title{
De-blending deep Herschel surveys: A multi-wavelength approach ${ }^{\star}$
}

\author{
W. J. Pearson ${ }^{1,2}$, L. Wang ${ }^{1,2}$, F. F. S. van der Tak ${ }^{1,2}$, P. D. Hurley ${ }^{3}$, D. Burgarella ${ }^{4}$, and S. J. Oliver ${ }^{3}$ \\ 1 SRON Netherlands Institute for Space Research, Landleven 12, 9747 AD Groningen, The Netherlands \\ e-mail: w.j . pearson@sron.nl \\ 2 Kapteyn Astronomical Institute, University of Groningen, Postbus 800, 9700 AV Groningen, The Netherlands \\ 3 Astronomy Centre, Department of Physics and Astronomy, University of Sussex, Falmer, Brighton BN1 9QH, UK \\ 4 Aix-Marseille Université, CNRS, LAM (Laboratoire d'Astrophysique de Marseille) UMR 7326, 13388 Marseille, France
}

Received 21 November 2016 / Accepted 6 April 2017

\begin{abstract}
Aims. Cosmological surveys in the far-infrared are known to suffer from confusion. The Bayesian de-blending tool, XID+, currently provides one of the best ways to de-confuse deep Herschel SPIRE images, using a flat flux density prior. This work is to demonstrate that existing multi-wavelength data sets can be exploited to improve XID+ by providing an informed prior, resulting in more accurate and precise extracted flux densities.

Methods. Photometric data for galaxies in the COSMOS field were used to constrain spectral energy distributions (SEDs) using the fitting tool CIGALE. These SEDs were used to create Gaussian prior estimates in the SPIRE bands for XID+. The multi-wavelength photometry and the extracted SPIRE flux densities were run through CIGALE again to allow us to compare the performance of the two priors. Inferred ALMA flux densities $\left(F_{\mathrm{ALMA}}^{\mathrm{infer}}\right)$, at $870 \mu \mathrm{m}$ and $1250 \mu \mathrm{m}$, from the best fitting SEDs from the second CIGALE run were compared with measured ALMA flux densities $\left(F_{\mathrm{ALMA}}^{\mathrm{mea}}\right)$ as an independent performance validation. Similar validations were conducted with the SED modelling and fitting tool MAGPHYS and modified black-body functions to test for model dependency. Results. We demonstrate a clear improvement in agreement between the flux densities extracted with XID+ and existing data at other wavelengths when using the new informed Gaussian prior over the original uninformed prior. The residuals between $F_{\text {ALMA }}^{\text {meas }}$ and $F_{\mathrm{ALMA}}^{\mathrm{infer}}$ were calculated. For the Gaussian priors these residuals, expressed as a multiple of the ALMA error $(\sigma)$, have a smaller standard deviation, $7.95 \sigma$ for the Gaussian prior compared to $12.21 \sigma$ for the flat prior; reduced mean, $1.83 \sigma$ compared to $3.44 \sigma$; and have reduced skew to positive values, 7.97 compared to 11.50 . These results were determined to not be significantly model dependent. This results in statistically more reliable SPIRE flux densities and hence statistically more reliable infrared luminosity estimates.
\end{abstract}

Key words. galaxies: statistics - infrared: galaxies

\section{Introduction}

Infrared (IR) radiation makes up approximately half of the total extragalactic emission that we observe (e.g. Dole et al. 2006; Burgarella et al. 2013). As a result, it is important to observe at these wavelengths to gain a better understanding of our Universe. The ESA Herschel Space Observatory (Pilbratt et al. 2010) Spectral and Photometric Imaging Receiver (SPIRE; Griffin et al. 2010) cosmological surveys probe the far-IR but are known to be affected by source confusion (e.g. Nguyen et al. 2010; Oliver et al. 2012), with object separation in the COSMOS2015 catalogue (Laigle et al. 2016) $\lesssim 8^{\prime \prime}$ and the SPIRE smallest beam size of $18^{\prime \prime}$ (Griffin et al. 2010). There have been a number of tools created to de-blend SPIRE images, such as DESPHOT (Roseboom et al. 2010, 2012; Wang et al. 2014) or T-PHOT (Merlin et al. 2015). These tools mostly use maximum likelihood estimation to generate flux density estimates using galaxy positions extracted from a shorter wavelength image.

More recently, the probabilistic, Bayesian de-blender XID+ (Hurley et al. 2017) has been developed, overcoming the main weaknesses of DESPHOT, namely its tendency to assign all the

* Herschel is an ESA space observatory with science instruments provided by European-led Principal Investigator consortia and with important participation from NASA. flux density to one source when many sources are within a single beam, as well as the poor estimation of variance and co-variance of sources. This is achieved by exploring the full posterior distribution (see Sect. 3.1.1) using the Bayesian statistical inference tool Stan (Carpenter et al. 2017). As a result of posterior exploration, XID+ produces much better flux density precision with more realistic associated uncertainties than DESPHOT across all three SPIRE bands (Hurley et al. 2017). However, the current, publicly available XID+ only uses a flat and uninformed flux density prior.

The aim of this paper is to show how the performance of XID+ can be advanced by exploiting the large amount of multi-wavelength data available in the popular COSMOS field (Scoville et al. 2007) as part of the continuing development of XID+, although the technique can be applied in any deep field with multi-wavelength data available. Throughout this paper, Wilkinson Microwave Anisotropy Probe year 7 cosmology (Komatsu et al. 2011; Larson et al. 2011) is followed: $\Omega_{\mathrm{M}}=$ $0.27, \Omega_{\Lambda}=0.73$, and $H_{0}=70.4 \mathrm{~km} \mathrm{~s}^{-1} \mathrm{Mpc}^{-1}$.

\section{Data}

For this work, a large multi-wavelength data set from a field covered by the Herschel Multi-tiered Extragalactic 
Survey (HerMES; Oliver et al. 2012) was required. The multiwavelength data is needed to generate flux density priors for deblending the SPIRE images from HerMES. A secondary data set is also required for validation of the extracted flux densities. For this we chose data from the Atacama Large Millimeter/submillimeter Array (ALMA), due to its higher resolution and because the $850 \mu \mathrm{m}$ and $1100 \mu \mathrm{m}$ bands are dominated by the same emission as the SPIRE bands (e.g. Scoville et al. 2014, 2016).

Thermal emission dominates at wavelengths of approximately $50 \mu \mathrm{m}$ or more and contains the Rayleigh-Jeans (RJ) tail from approximately $100 \mu \mathrm{m}$ to $1100 \mu \mathrm{m}$ in the rest frame (e.g. Draine 2003; Draine \& Li 2007). Thus, at least one SPIRE band lies within the RJ tail up to redshifts of 4 and all three SPIRE bands are dominated by thermal emission at all redshifts studied here. Similarly, the $870 \mu \mathrm{m}$ ALMA band remains in the RJ tail for the entirety of this study, while the $1250 \mu \mathrm{m}$ ALMA band enters the RJ region at redshifts of 0.14 and so was chosen for high redshift objects only. Evidently, the SPIRE bands and the two ALMA bands chosen for this study are both dominated by the same physical processes, certainly up to redshifts of at least 4, making ALMA data a good choice to validate the SPIRE data.

The COSMOS field was chosen because of the prevalence of multi-wavelength data, and because the SPIRE images within COSMOS are considered to be reasonably homogenous and of high quality (see Appendix A). The COSMOS2015 catalogue (Laigle et al. 2016) was used as an ancillary data set as it contains data on over 1.2 million objects in over 30 wavelength bands. For this work, only bands from the ultraviolet to the midIR were used. The Subaru narrow bands were omitted as similar wavelengths are covered by the Subaru intermediate bands ${ }^{1}$. The Spitzer Multiband Imaging Photometer (Rieke et al. 2004) $24 \mu \mathrm{m}$ data were also not used as they are also noticeably affected by confusion, although not to the same extent as the SPIRE data (e.g. Dole et al. 2004; Rieke et al. 2004). It also lies in a region of the spectrum that is more complicated to model, with contributions from active galactic nuclei (AGN), thermal dust emission and non-thermal polycyclic aromatic hydrocarbons. The $3^{\prime \prime}$ aperture data were used, where available, to match the Spitzer Infrared Array Camera data.

The latest SPIRE maps for the COSMOS field, Data Release 4 from HerMES (Oliver et al. 2012), from the Herschel Database in Marseille ${ }^{2}$ were used for the SPIRE extraction. These maps have a beam size of $18.1^{\prime \prime}, 25.2^{\prime \prime}$, and $36.6^{\prime \prime}$ and $5 \sigma$ confusion limits of $24.0,27.5$, and $30.5 \mathrm{mJy}$ for the $250 \mu \mathrm{m}$, $350 \mu \mathrm{m}$, and $500 \mu \mathrm{m}$ bands, respectively (Griffin et al. 2010; Nguyen et al. 2010).

The ALMA archive ${ }^{3}$ was searched for objects within the COSMOS field with positive flux densities at $870 \mu \mathrm{m}$ or $1250 \mu \mathrm{m}$. A total of 214 objects were found that met these criteria: 192 objects with $870 \mu \mathrm{m}$ data at $z<3$ from Scoville et al. $(2014,2016)$ and 22 objects with $1250 \mu \mathrm{m}$ data at $z>3$ from Scoville et al. (2016). In the original studies, these objects were selected as they have stellar masses of approximately $10^{11} \mathrm{M}_{\odot}$. Of the 214 objects, $43(20 \%)$ have a signal-to-noise ratio of less than $2 \sigma$, all of which are from Scoville et al. (2014), due to the higher values of $1 \sigma \mathrm{rms}$ noise. The reader is referred to Scoville et al. (2014) and Scoville et al. (2016) for discussions on the signal-to-noise of the ALMA observations.

\footnotetext{
1 The Subaru narrow bands are used in the photometric redshift derivation in the COSMOS2015 catalogue.

2 http://hedam. lam. fr

3 https://almascience.nrao.edu/alma-data/archive
}

The ALMA data were matched to the COSMOS2015 sources. With a positional accuracy of $0.15^{\prime \prime}$ for the COSMOS2015 catalogue (Laigle et al. 2016) and a pointing accuracy of $0.6^{\prime \prime}$ for ALMA, matching within $1^{\prime \prime}$ of the ALMA sources was deemed adequate. For one ALMA object there was more than one match to the COSMOS2015 catalogue, so the closest object was used. Once matched, the photometric redshifts from COSMOS2015 were used.

\section{Methodology}

\subsection{Tools}

\subsubsection{XID+}

XID $+{ }^{4}$ (Hurley et al. 2017) is a probabilistic de-blending tool created to extract source flux densities from photometry maps that are affected by source confusion. This is achieved by using Bayesian inference to explore the posterior distribution function. Once converged, the flux density is reported along with the upper and lower $1 \sigma$ uncertainties. In the original version, XID+ uses a flat prior in parameter space, between zero and the brightest value in the map, along with the source positions on the sky. This work introduces a more informed Gaussian prior, again truncated between zero and the brightest value in the map. The mean and sigma for these Gaussian priors are generated by using CIGALE spectral energy distribution (SED) models to estimate the flux densities for the mean and using twice the error on these estimates as the sigma, to be conservative (see also Sects. 3.1.2 and 3.2).

To allow parallelisation, which reduces the time XID+ takes to de-blend the map, the map is split into tiles based on the Hierarchical Equal Area isoLatitude Pixelization of a sphere (HEALPix; Górski et al. 2005) system using order 11, which corresponds to an area of $2.95 \operatorname{arcmin}^{2}$ per tile. Order 11 was chosen as it is a compromise between the number of objects in a tile - more objects means a more reliable flux density extraction - and the time it takes a tile to run; here a tile was required to have a run time of less than one week. XID+ was run using four cores per tile on the 162 node Peregrine high performance computing cluster (HPC) at the University of Groningen.

\subsubsection{CIGALE}

Code Investigating GALaxy Emission ${ }^{5}$ (CIGALE; Noll et al. 2009) is a SED modelling and fitting tool with an improved fitting procedure by Serra et al. (2011). Here, the Python version 0.9.0 is used (Boquien et al., in prep.; Burgarella et al., in prep.) to generate SEDs and fit them to the data from COSMOS2015 to estimate the SPIRE $250 \mu \mathrm{m}, 350 \mu \mathrm{m}$, and $500 \mu \mathrm{m}$ flux densities. CIGALE was run using one node of the Peregrine HPC.

CIGALE models are based around three main components: stars, dust, and AGN. The SEDs generated by CIGALE used our choice of a double exponentially declining star formation history (SFH), Bruzual \& Charlot (2003) stellar emission, Chabrier (2003) initial mass function, Calzetti et al. (2000) dust attenuation, the Draine et al. (2014) update of the Draine \& Li (2007) IR dust emission, and Fritz et al. (2006) AGN models. A list of the parameters used, where they differ from the default values, can be found in Appendix B, along with a brief justification.

\footnotetext{
4 https://github.com/H-E-L-P/XID_plus

5 http://cigale.lam.fr/
} 


\subsection{Extracting the SPIRE flux densities}

CIGALE and XID+ were used to extract the flux densities in the SPIRE bands. The ALMA data were not used in this process. The pipeline begins by using the multi-wavelength data from COSMOS2015 to generate estimates for the SPIRE flux densities and uncertainties using CIGALE. Approximately $1.3 \%$ of the objects in the COSMOS2015 catalogue have no known redshift. These objects were removed from the CIGALE run and assigned an arbitrary flux density of $7 \mathrm{mJy}$, along with an artificially large error to produce a functionally flat prior. A further $2.9 \%$ of the COSMOS2015 catalogue objects are classified as non-galaxies. These objects can be run through CIGALE, but care must be taken; they will be assigned flux density estimates with the assumption that they have the SED of a galaxy. To compensate for this, the errors were artificially inflated to create an almost flat prior. The objects with no redshift or those classified as non-galaxies cannot simply be discarded as they will likely appear in the SPIRE images. For the remaining objects in the COSMOS2015 catalogue with redshifts and classified as galaxies, the errors were multiplied by two to prevent overconstraint in XID+ (see also Sect. 4.1).

The SPIRE estimates from CIGALE, along with the added data for objects without redshifts, were used as the means in the priors for XID+, while the expanded errors were used as the standard deviations. XID+ was then run on the SPIRE images. As the priors for the objects that are not galaxies, or have no redshift, are effectively flat, the flux for these objects is free to change such that it will not interfere with the fitting of the more constrained sources.

For the flat prior flux densities, the tiles of interest were run through XID+ with a flat flux density prior. These flat prior flux densities were compared to the informed prior flux densities.

\section{Results}

\subsection{CIGALE and XID+ performance}

To check whether the CIGALE predictions for the SPIRE sources are reasonable, the flux densities for the 100 brightest sources at $250 \mu \mathrm{m}$ from the COSMOS2015 catalogue, which also have detections at 350 and $500 \mu \mathrm{m}$, were compared to the predicted flux densities from CIGALE. Blind extraction has been shown to overestimate $250 \mu \mathrm{m}$ flux densities by up to $150 \%$, even for bright sources above the $5 \sigma$ confusion limit (Scudder et al. 2016). Thus, we expect the flux densities from CIGALE to be systematically lower than those in COSMOS2015. We note, however, that the COSMOS2015 catalogue has SPIRE data extracted using the previous generation deblending tool DESPHOT, not blind extraction, so the overestimation will not be the same, but a comparison is still valid (Scudder 2017, priv. comm.). It is also possible for the CIGALE predictions to underpredict for some sources, but this is accounted for in our increased error (see below).

Figure 1 shows that the CIGALE SPIRE predictions are indeed lower than the COSMOS2015 flux densities: the blue data points for the $250 \mu \mathrm{m}$ sources fall below the magenta one-to-one line. The cyan line is the locus where the CIGALE flux density is $40 \%$ the COSMOS2015 flux density: the fraction that is expected if the catalogue results are overestimated by $150 \%$. This line splits the $250 \mu \mathrm{m}$ data approximately in half, with $52 \mathrm{ob}-$ jects below the line and 48 above, which would be expected if the catalogue results are $150 \%$ too high. However, there is a large scatter. The $350 \mu \mathrm{m}$ and $500 \mu \mathrm{m}$ bands are less well split, with 59

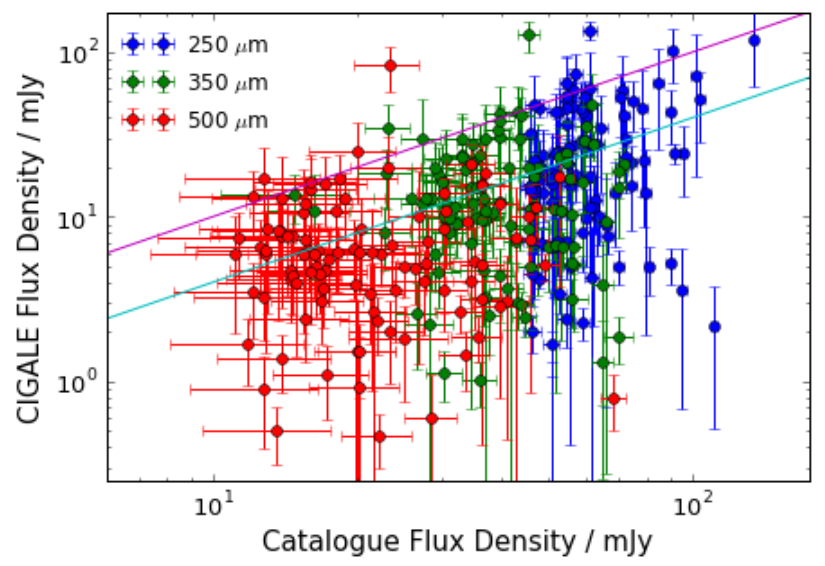

Fig. 1. Scatter plot of the predicted CIGALE flux densities against the flux densities from the COSMOS2015 catalogue for the $250 \mu \mathrm{m}$ (blue points), $350 \mu \mathrm{m}$ (green points), and $500 \mu \mathrm{m}$ (red points). The errors for the catalogue flux are instrumental noise plus confusion noise. The oneto-one line (magenta) and $y=0.4 x$ (cyan) illustrate that the data better fits the idea that bright sources in catalogues are overestimated.

and 68 objects below the cyan line, respectively. With the lower resolution of the $350 \mu \mathrm{m}$ and $500 \mu \mathrm{m}$ bands, it is likely that multiplicity will have a greater impact, so it is not surprising that the longer wavelengths have more objects with predicted fluxes below the cyan line.

We can also calculate how many of the 100 bright objects have CIGALE flux densities consistent with $40 \%$ of the catalogue flux densities within CIGALE errors. This is 39, 44, and 45 for the $250 \mu \mathrm{m}, 350 \mu \mathrm{m}$, and $500 \mu \mathrm{m}$ bands, respectively. If the error is doubled the number of objects increases to 65,70 , and 64 for the $250 \mu \mathrm{m}, 350 \mu \mathrm{m}$, and $500 \mu \mathrm{m}$ bands and is consistent with what is expected for a $1 \sigma$ uncertainty. Therefore, we decided to expand the errors from CIGALE by a factor of two.

To check that the results from XID+ with an informed prior are not just the prior itself, Fig. 2 shows a plot of the extracted $250 \mu \mathrm{m}$ flux densities using the informed Gaussian against the $250 \mu \mathrm{m}$ flux density priors from CIGALE for both the ALMA selected objects (dark blue) as well as the full probability density function derived from the posterior samples (fPDF) of all 63701 objects in the same tiles as the ALMA objects, from high (light red) to low (dark red). If XID+ was simply returning the prior, all the points would lie close to or along the red one-to-one line. This is evidently not the case for the ALMA objects, with extracted flux densities up to 1.5 dex away from their flux density priors, or for the fPDF. The $350 \mu \mathrm{m}$ and $500 \mu \mathrm{m}$ results are similar.

\subsection{Comparison of ALMA data to CIGALE SEDs}

The SPIRE data constrain the peak of the cold dust emission in a galaxy's SED and, if extracted correctly, any data in the long wavelength tail should lie on the SED. To test this, the SPIRE data from XID+, along with the data from the COSMOS2015 catalogue, were run through CIGALE to create the best fitting SED for all of the data. One object failed to converge, resulting in no output from CIGALE, and so had to be omitted. ALMA data were then compared to the best fitting SEDs.

For the 213 objects that successfully ran through CIGALE, the residuals between the measured ALMA flux densities and the ALMA flux densities inferred from the best fitting SEDs were calculated. These residuals $(\gamma)$ are presented in Table 1 


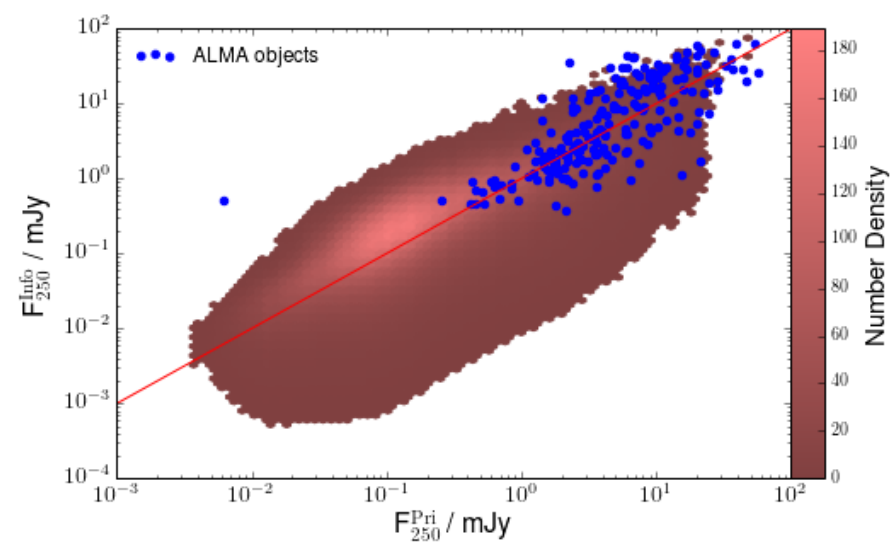

Fig. 2. Extracted $250 \mu \mathrm{m}$ flux densities using the informed Gaussian $\left(F_{250}^{\text {Info }}\right)$ against the $250 \mu \mathrm{m}$ flux density priors from CIGALE $\left(F_{250}^{\mathrm{Pri}}\right)$ for the ALMA selected sources (blue). The fPDF of all objects extracted from the same tiles as the ALMA sources are also shown and presented from high (light red) to low (dark red) density. The red line is the oneto-one line.

Table 1. Absolute residuals $(|\gamma|)$ between the measured ALMA flux densities and the fluxes of the best fitting SEDs at the ALMA wavelengths expressed as a multiple of the error on the ALMA flux density.

\begin{tabular}{ccc}
\hline \hline$|\gamma|$ & Flat prior & Informed prior \\
\hline 1 & $77(36.2 \%)$ & $98(46.0 \%)$ \\
2 & $121(56.8 \%)$ & $142(66.7 \%)$ \\
3 & $146(68.5 \%)$ & $166(77.9 \%)$ \\
4 & $160(75.1 \%)$ & $184(86.4 \%)$ \\
5 & $177(83.1 \%)$ & $192(90.1 \%)$ \\
\hline
\end{tabular}

Notes. The values in Cols. 2 and 3 are the number (percentage) of objects with a $|\gamma|$ less than the value in Col. 1 .

and Fig. 3 as multiples of the errors on the ALMA flux densities $(\sigma)$, i.e. $\gamma=\operatorname{residual} / \sigma$. A positive $\gamma$ means that the measured ALMA flux density is greater than the inferred flux density, and the closer the value of $\gamma$ is to zero, the better XID+ had performed.

Figure 3 demonstrates the improvement that the informed prior gives over the flat prior. The informed Gaussian prior has a reduced spread around zero, a standard deviation of $7.95 \sigma$ compared to $12.21 \sigma$ for the flat prior, indicating that the informed prior is providing a better fit to the ALMA data than the flat prior. A reduced skew for the informed prior with a skew of 7.97 with respect to the flat prior with a skew of 11.50 , and a reduction in the mean of the distribution from $3.44 \sigma$ for the flat prior to $1.83 \sigma$ for the informed prior also demonstrates improved performance. With the reduced skew and lower mean, XID+ does not appear to underpredict the SPIRE flux densities as often with the informed prior. Table 1 shows the number of objects that have an absolute value of $\gamma(|\gamma|)$ below a certain threshold. The number of objects below each $|\gamma|$ value is greater for the informed prior than it is for the flat prior, demonstrating that there is better agreement between the inferred ALMA flux densities of the best fitting SEDs and the measured data when using the informed prior than when using the flat prior. There is an increase of $27.3 \%$ in the number of sources at $|\gamma|<1$ when using the informed prior over the flat prior. This reduces to $8.5 \%$ at $|\gamma|<5$ and gives an average increase of $16.4 \%$ in the number of sources across all five ranges. The maximum $|\gamma|$ values follow the same trend with the flat prior giving a maximum $|\gamma|$ of 167.304 compared to 95.180 for the

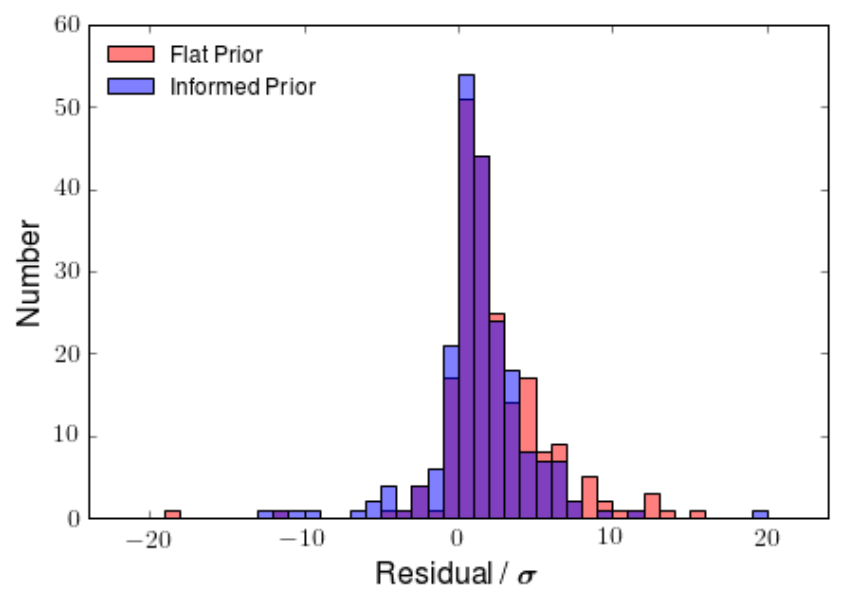

Fig. 3. Distribution of the residuals between the measured ALMA flux densities and the flux densities inferred from the SEDs at the ALMA wavelengths expressed as a multiple of the error on the ALMA flux density $(\sigma)$ for the flat prior (red) and the informed Gaussian prior (blue). The informed prior reduces the mean from $3.44 \sigma$ to $1.83 \sigma$, the standard deviation from $12.21 \sigma$ to $7.95 \sigma$, and the skew from 11.50 to 7.97 .

informed prior. The minimum $|\gamma|$ for the flat prior is that same as that of the informed prior.

The ALMA objects can be split into three groups: Group A, where the informed Gaussian prior provides a $|\gamma|$ value more than $5 \%$ smaller than the flat prior $|\gamma|$; Group B, where the informed prior $|\gamma|$ is within $5 \%$ of the flat prior $|\gamma|$; and Group C, where the informed $|\gamma|$ is more than $5 \%$ larger than the flat $|\gamma|$. Figure $4 \mathrm{a}$ provides an example of one of the 99 Group A objects. It can clearly be seen that the SED for the informed prior (blue line) is in much better agreement with the measured ALMA flux density (green point) than the SED for the flat prior (red line). There are 70 objects in Group B while Group C contains the remaining 44 objects, examples of which can be found in Figs. $4 \mathrm{~b}$ and c, respectively. With Group A being the largest group, it is evident that the informed prior produces more accurate results than the flat prior. If the objects with less than 2 ALMA signal-to-noise (see Sect. 2) are removed, the number of objects in Groups A, $\mathrm{B}$, and $\mathrm{C}$ are 89,51 , and 35 , respectively. Thus, the removal of these objects does not change the conclusion that, on average, the informed prior produces more accurate results.

To see how the two XID+ priors compare with each other, Fig. 5 shows the ratio between the flux density extracted using the informed prior $\left(F_{250}^{\text {Info }}\right)$ and flat prior $\left(F_{250}^{\text {Flat }}\right)$ against $F_{250}^{\text {Info }}$. The

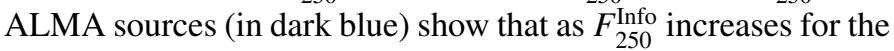
ALMA objects, the ratio $F_{250}^{\text {Info }} / F_{250}^{\text {Flat }}$ increases, becoming unity at $F_{250}^{\text {Info }} \approx 3 \mathrm{mJy}$, with smaller ratios below this flux density and higher ratios above. This implies that the flat prior overestimates flux densities for objects fainter than $3 \mathrm{mJy}$ and underestimates flux densities for objects brighter than $3 \mathrm{mJy}$, with respect to the informed prior. This trend also appears for the fPDF, shown from high (light red) to low (dark red). There is an indication that the relation begins to flatten for high flux densities, which is expected as both priors should perform equally well at higher flux densities. However, $F_{250}^{\text {Info }} / F_{250}^{\text {Flat }}$ being above unity is surprising. This may be the high source density affecting the flat prior results: the flat prior may be "too democratic" as it assigns flux density to each object and so assigns too much to the faint objects and too little to the bright objects.

Figure 6 illustrates how the difference between the flat and informed Gaussian XID+ priors affects the IR luminosity $\left(L_{\mathrm{IR}}\right)$. 


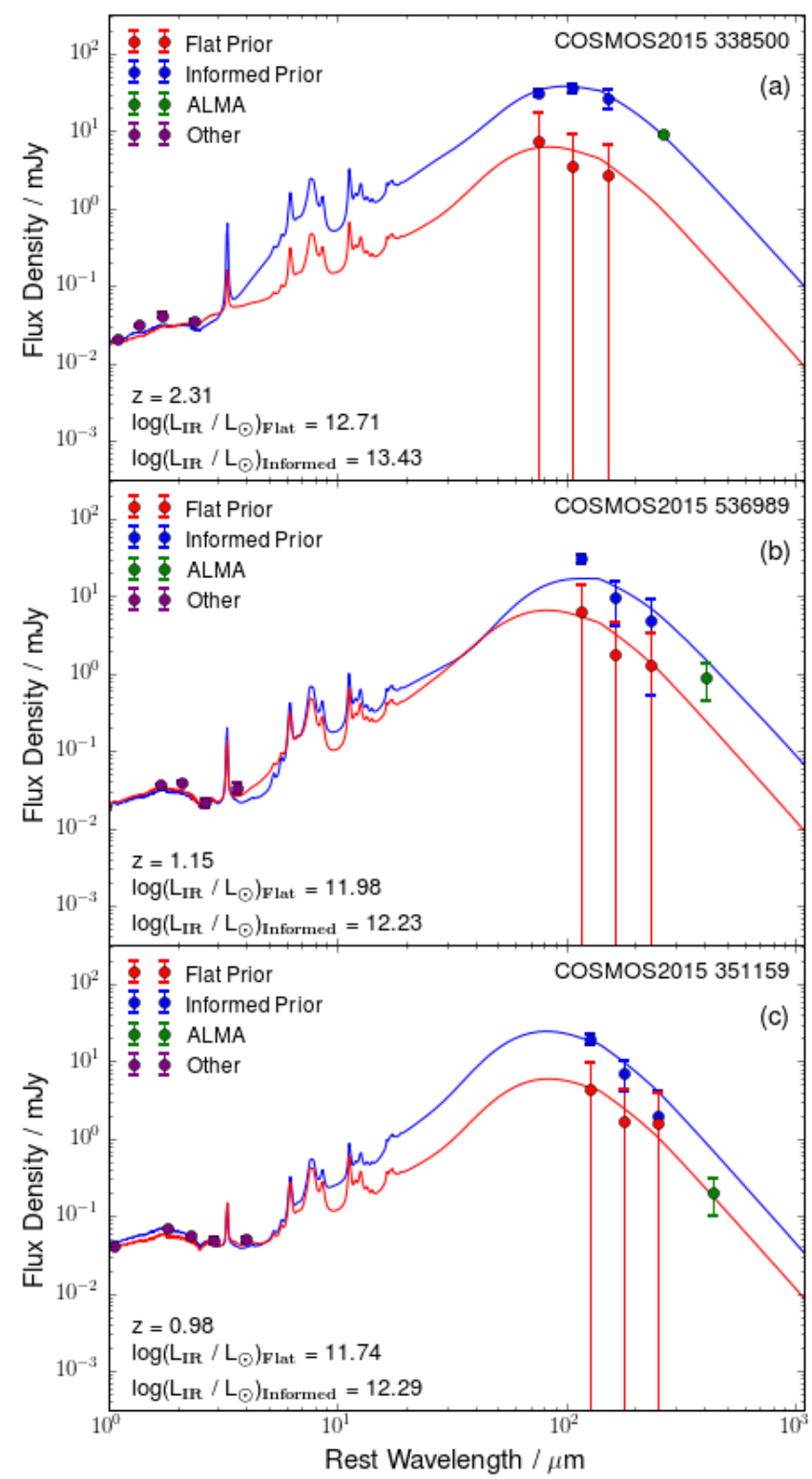

Fig. 4. Example plots showing the best fitting SEDs (curves) for the extracted SPIRE flux densities (red and blue points) using the flat prior (red) and informed Gaussian prior (blue). The other multiwavelength data from the COSMOS2015 catalogue (purple) are overplotted. Panel a) is an example of Group A, illustrating how the informed prior can increase the agreement between the best fitting SED and the measured ALMA flux density (green). Panel b) is for Group B, illustrating how the informed and flat priors can give equal agreement between the best fitting SED and the measured ALMA flux density. Panel c) is for Group C, showing how the flat prior can occasionally give better agreement.

The $L_{\mathrm{IR}}$ was calculated by integrating the best fitting SED between 3 and $1100 \mu \mathrm{m}$ at rest. For the low $(z<1)$ and high $(z>3)$ redshift objects, the change in prior has little effect on $L_{\mathrm{IR}}$. However, for the intermediate redshift $(1<z<3)$ objects, there appears to be an increase in $L_{\mathrm{IR}}$, although the number of objects in the sample is too small to draw any robust conclusions. As can be seen in the right panel of Fig. 6, this results in a smoother distribution of luminosities across the sample presented. For comparison, the green line in Fig. 6 shows the $5 \sigma$ confusion limit for the $250 \mu \mathrm{m}$ band, calculated by scaling the Wuyts et al. (2008) SED template to the $250 \mu \mathrm{m} 5 \sigma$ confusion

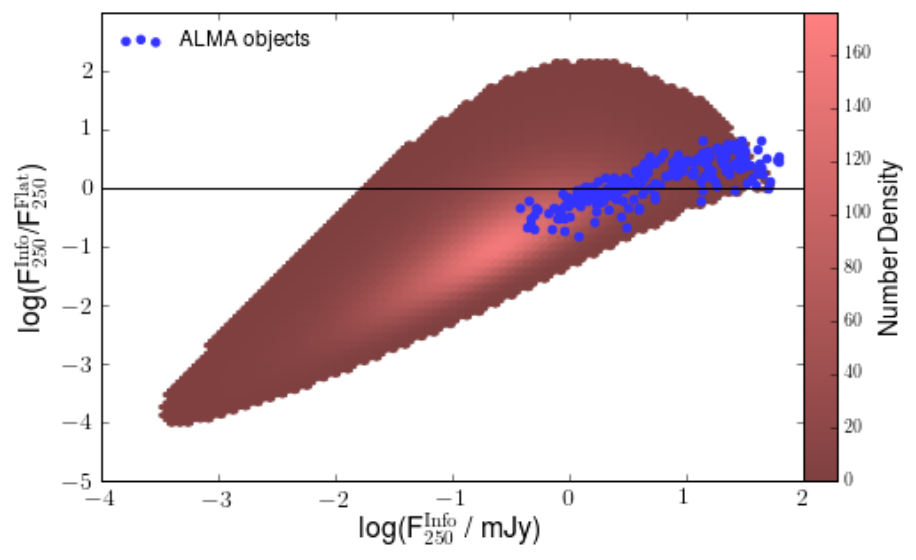

Fig. 5. Ratio of the $250 \mu \mathrm{m}$ flux densities extracted using the informed Gaussian prior $\left(F_{250}^{\text {Info }}\right)$ to those extracted with the flat prior $\left(F_{250}^{\text {Flat }}\right)$ against $\left(F_{250}^{\text {Info }}\right.$. The ALMA sources are in dark blue, while the statistical average number density is from light red (high) to dark red (low).

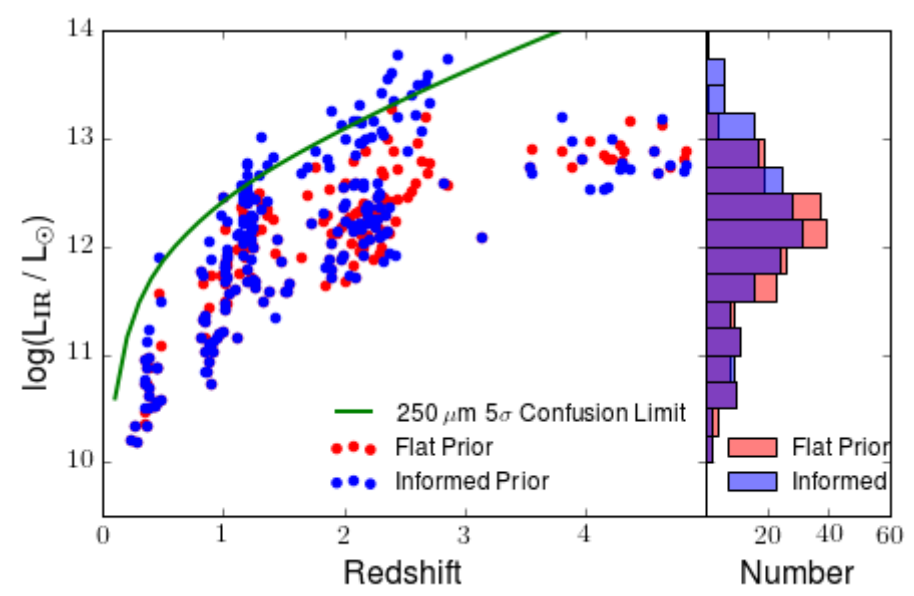

Fig. 6. Scatter plot of the infrared (IR, 3-1100 $\mu \mathrm{m}$ ) luminosity vs. redshift for the results from XID+ with the flat prior (red points/bars) and the informed Gaussian prior (blue points/bars) with a histogram of the IR luminosities. The green line corresponds to the $250 \mu \mathrm{m} 5 \sigma$ confusion limit.

limit of $24.0 \mathrm{mJy}$ and integrating between 3 and $1100 \mu \mathrm{m}$ for a range of redshifts. Twenty of the objects with an informed prior, and one with a flat prior, do not fall below this confusion limit.

\subsection{Model dependance}

To check whether the difference between the informed prior and flat prior results is caused by our CIGALE model, we also fitted the data from the COSMOS2015 catalogue and the extracted SPIRE data for the 213 ALMA sources with MAGPHYS $^{6}$ (da Cunha et al. 2008), and we fitted modified blackbody (MBB) functions to the SPIRE data from the 213 sources. MAGPHYS is a SED modelling and fitting tool that uses different models from CIGALE, while MBBs are commonly used to model thermal dust emission (e.g. Viero et al. 2013; Casey et al. 2014; Wang et al. 2016). Here we use a MBB of the form

$S_{v} \propto v^{\beta} \times B_{v}(T)$

6 http://www . iap.fr/magphys/magphys/MAGPHYS .html 
Table 2. Absolute residuals $(|\gamma|)$ between the measured ALMA flux densities and the fluxes of the best fitting MBB templates and MAGPHYS SEDs at the ALMA wavelengths expressed as a multiple of the error on the ALMA flux density.

\begin{tabular}{lccc}
\hline \hline Model & $|\gamma|$ & Flat prior & Informed prior \\
\hline MBB & 1 & $62(29.1 \%)$ & $70(32.9 \%)$ \\
& 2 & $106(49.8 \%)$ & $120(56.3 \%)$ \\
& 3 & $134(62.9 \%)$ & $155(72.8 \%)$ \\
& 4 & $152(71.4 \%)$ & $171(80.3 \%)$ \\
& 5 & $168(78.9 \%)$ & $181(85.0 \%)$ \\
\hline MAGPHYS & 1 & $72(33.8 \%)$ & $79(37.1 \%)$ \\
& 2 & $119(55.9 \%)$ & $132(62.0 \%)$ \\
& 3 & $138(64.8 \%)$ & $160(75.1 \%)$ \\
& 4 & $156(73.2 \%)$ & $169(79.3 \%)$ \\
& 5 & $171(80.3 \%)$ & $177(83.1 \%)$ \\
\hline
\end{tabular}

Notes. The values in Cols. 3 and 4 are the number (percentage) of objects with a $|\gamma|$ less than the value in Col. 2 .

where $B_{v}(T)$ is the black-body function for dust at temperature $T$. The emissivity $\beta$ was fixed at 1.5 , and $T$ was allowed to vary between $10 \mathrm{~K}$ and $150 \mathrm{~K}$. With these results, we recreated Table 1 for the MBB and MAGPHYS results, and examined the populations of the three groups, A, B, and C.

For the MBB, qualitatively the results are the same, although the division between the flat and informed priors is less wide. The results, shown in Table 2, show that the number of objects within certain $|\gamma|$ thresholds is greater for the informed prior than for the flat prior, supporting the idea that the results from XID+ using the informed prior are more reliable than the results with the flat prior. There is an average increase of $12.4 \%$ in the number of sources across all five bins, which is less than the average over all five bins when compared with CIGALE. Also, looking at the distribution of objects in the three groups, we find that Group A again has the most objects (126), while Groups B and $\mathrm{C}$ have fewer (37 and 51 objects, respectively).

As with the results from the MBB, the results from MAGPHYS also have the same qualitative results as those using CIGALE. For MAGPHYS, the data from COSMOS2015 along with the extracted flux densities for the SPIRE bands were used, and SEDs were fitted to these data. Similar to CIGALE, the flux densities at $870 \mu \mathrm{m}$ and $1250 \mu \mathrm{m}$ were extracted from the best fitting SEDs and compared to the ALMA data. The results are presented in Table 2 and again show a greater number of objects within the values of $|\gamma|$ examined for the informed prior with respect to the flat prior, with an average increase of $9.7 \%$ in the number of sources across all five $|\gamma|$ ranges. As with the CIGALE and MBB results, Group A again contains the greatest number of objects (108), followed by Group C (65), and Group B (41). As both the MAGPHYS and the MBB results are consistent with the CIGALE results, we believe that there is little model dependence in our conclusions.

\section{Conclusions}

$\mathrm{XID}+$ is one of the most advanced de-blending tools available. Here, we extend the flux density prior from a simple flat prior (Hurley et al. 2017) and explore the idea of using supplementary data to provide a more physically motivated flux density prior. Using a rich set of multi-wavelength data, an informed Gaussian prior was introduced to XID+ and applied to 214 objects with
ALMA detections in the COSMOS field. Using this method, it was shown that the agreement between the measured ALMA flux densities and the inferred flux densities (calculated with CIGALE) at the ALMA wavelengths improved by an average of $16.4 \%$ in the number of objects in each of the five $|\gamma|$ ranges examined in Sect. 4. If all three models used for the ALMA comparison are considered, there is an average increase of $12.8 \%$ demonstrating that, qualitatively, the results are not model dependent. However, the exact quantitative improvement seen is model dependent. This demonstrates that utilising supplementary data to provide a more physically motivated prior results in extracted flux density values that have improved agreement with data at other wavelengths.

Acknowledgements. We would like to thank the anonymous referee whose comments greatly improved this paper. We would like to thank the Center for Information Technology of the University of Groningen for their support and for providing access to the Peregrine high performance computing cluster. This research has made use of data from HerMES project (http://hermes.sussex.ac. uk/). HerMES is a Herschel Key Programme utilising Guaranteed Time from the SPIRE instrument team, ESAC scientists, and a mission scientist. The HerMES data were accessed through the Herschel Database in Marseille (HeDaM - http://hedam. lam.fr) operated by CeSAM and hosted by the Laboratoire d'Astrophysique de Marseille. HerMES DR4 was made possible through support of the Herschel Extragalactic Legacy Project, HELP (http://herschel. sussex.ac.uk). The research leading to these results has received funding from the European Union Seventh Framework Programme FP7/2007-2013/ under grant agreement No. 607254. This publication reflects only the author's view and the European Union is not responsible for any use that may be made of the information contained therein.

\section{References}

Bruzual, G., \& Charlot, S. 2003, MNRAS, 344, 1000

Burgarella, D., Buat, V., Gruppioni, C., et al. 2013, A\&A, 554, A70 Calzetti, D., Armus, L., Bohlin, R. C., et al. 2000, ApJ, 533, 682 Carpenter, B., Gelman, A., Hoffman, M., et al. 2017, J. Stat. Software, 76, 1 Casey, C. M., Narayanan, D., \& Cooray, A. 2014, Phys. Rep., 541, 45 Chabrier, G. 2003, PASP, 115, 763

Ciesla, L., Charmandaris, V., Georgakakis, A., et al. 2015, A\&A, 576, A10 da Cunha, E., Charlot, S., \& Elbaz, D. 2008, MNRAS, 388, 1595 Dole, H., Rieke, G. H., Lagache, G., et al. 2004, ApJS, 154, 93 Dole, H., Lagache, G., Puget, J.-L., et al. 2006, A\&A, 451, 417 Draine, B. T. 2003, ARA\&A, 41, 241

Draine, B. T., \& Li, A. 2007, ApJ, 657, 810

Draine, B. T., Aniano, G., Krause, O., et al. 2014, ApJ, 780, 172 Fritz, J., Franceschini, A., \& Hatziminaoglou, E. 2006, MNRAS, 366, 767 Górski, K. M., Hivon, E., Banday, A. J., et al. 2005, ApJ, 622, 759 Griffin, M. J., Abergel, A., Abreu, A., et al. 2010, A\&A, 518, L3 Hurley, P. D., Oliver, S., Betancourt, M., et al. 2017, MNRAS, 464, 885 Komatsu, E., Smith, K. M., Dunkley, J., et al. 2011, ApJS, 192, 18 Laigle, C., McCracken, H. J., Ilbert, O., et al. 2016, ApJS, 224, 24 Larson, D., Dunkley, J., Hinshaw, G., et al. 2011, ApJS, 192, 16 Merlin, E., Fontana, A., Ferguson, H. C., et al. 2015, A\&A, 582, A15 Mitchell, P. D., Lacey, C. G., Baugh, C. M., \& Cole, S. 2013, MNRAS, 435, 87 Nguyen, H. T., Schulz, B., Levenson, L., et al. 2010, A\&A, 518, L5 Noll, S., Burgarella, D., Giovannoli, E., et al. 2009, A\&A, 507, 1793 Oliver, S. J., Bock, J., Altieri, B., et al. 2012, MNRAS, 424, 1614 Pilbratt, G. L., Riedinger, J. R., Passvogel, T., et al. 2010, A\&A, 518, L1 Puglisi, A., Rodighiero, G., Franceschini, A., et al. 2016, A\&A, 586, A83 Rieke, G. H., Young, E. T., Engelbracht, C. W., et al. 2004, ApJS, 154, 25 Roseboom, I. G., Oliver, S. J., Kunz, M., et al. 2010, MNRAS, 409, 48 Roseboom, I. G., Ivison, R. J., Greve, T. R., et al. 2012, MNRAS, 419, 2758 Serra, P., Amblard, A., Temi, P., et al. 2011, ApJ, 740, 22 Scoville, N., Aussel, H., Brusa, M., et al. 2007, ApJS, 172, 1 Scoville, N., Aussel, H., Sheth, K., et al. 2014, ApJ, 783, 84 Scoville, N., Sheth, K., Aussel, H., et al. 2016, ApJ, 820, 83 Scudder, J. M., Oliver, S., Hurley, P. D., et al. 2016, MNRAS, 460, 1119 Viero, M. P., Wang, L., Zemcov, M., et al. 2013, ApJ, 772, 77 Wang, L., Viero, M., Clarke, C., et al. 2014, MNRAS, 444, 2870 Wang, L., Norberg, P., Gunawardhana, M. L. P., et al. 2016, MNRAS, 461, 1898 Wuyts, S., Labbé, I., Förster Schreiber, N. M., et al. 2008, ApJ, 682, 985 


\section{Appendix A: Homogeneity of COSMOS}

Figure A.1a shows the noise map for the 2 square degree $250 \mu \mathrm{m}$ SPIRE band in the COSMOS field with the area used for this study outlined in red and the position of the ALMA sources shown with blue points. As can be seen, the noise level within this area is approximately flat. For comparison, the noise map for the $250 \mu \mathrm{m}$ SPIRE band in the 11 square degree XMM-LSS field is shown in Fig. A.1b, which is much less homogenous. The noise maps for the $350 \mu \mathrm{m}$ and $500 \mu \mathrm{m}$ bands are very similar.
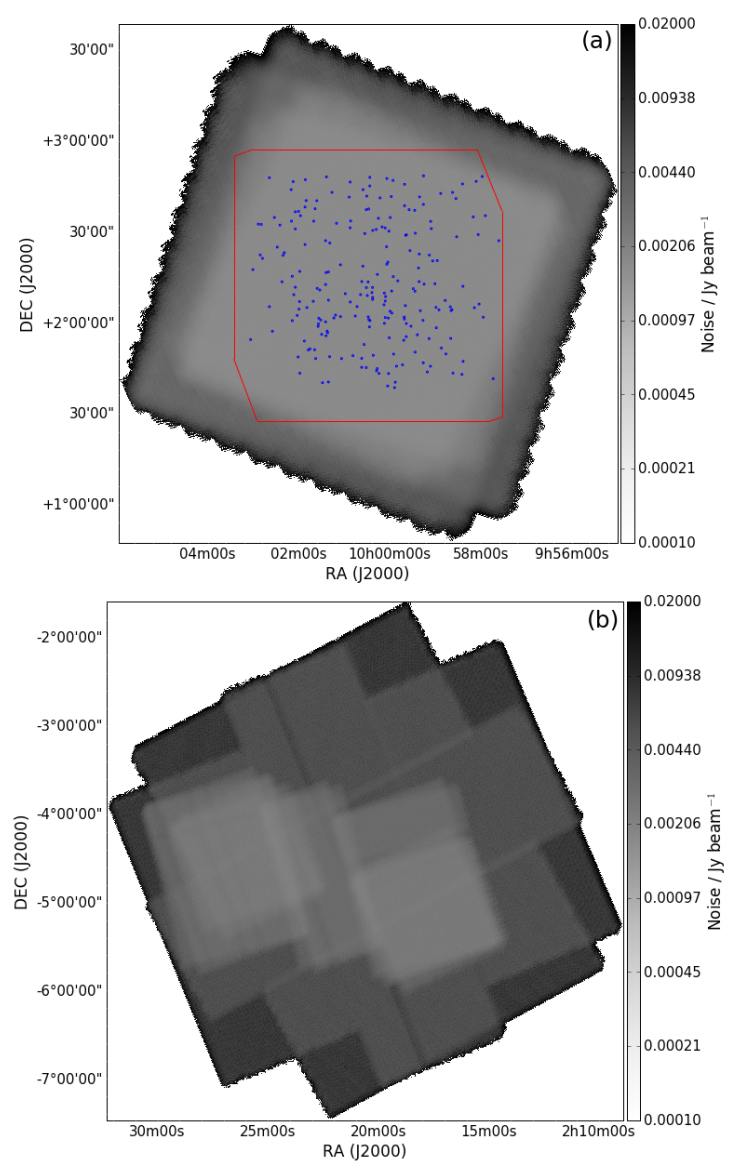

Fig. A.1. Noise map for the SPIRE $250 \mu \mathrm{m}$ band in the a) COSMOS field $\left(2 \mathrm{deg}^{2}\right)$ and b) XMM-LSS field $\left(11 \mathrm{deg}^{2}\right)$ from the HerMES DR4 (Oliver et al. 2012) and downloaded from HeDAM. For the COSMOS field, the outline of the study area is shown in red, while the ALMA sources are shown in blue.

\section{Appendix B: CIGALE parameters}

In this work, a double exponential SFH was used over the more commonly used exponentially declining or delayed exponential SFH as these two SFHs did not appear to reproduce the expected starburst population in the star formation rate vs. stellar mass plane. The e-folding time of the two stellar populations (old and young) in the SFH was roughly matched to that of Mitchell et al. (2013). As Mitchell et al. (2013) used a single declining exponential SFH, the e-folding times were split with the burst population taking values of $9 \mathrm{Gyr}$ and above and the main population taking values of less than 9 Gyr. For the ages of the main population, the values were sampled between 1 and 13 Gyr with a $\log$ distribution. The mass fraction of the burst population follows Ciesla et al. (2015) along with the age of the young stellar population, which also had a lower age of $0.001 \mathrm{Gyr}$ added. The Bruzual \& Charlot (2003) stellar population model was used with a Chabrier (2003) initial mass function. As this study was not designed to explore the metallicity of galaxies, it was decided to leave the metallically at solar.

The dust colour excess $\left(E(B-V)^{\star}\right)$ is often measured to be $\lesssim 0.6$, with a higher number of objects with lower excesses (e.g. Calzetti et al. 2000), so this region has been sampled for the SED models. However, it is possible that the $E(B-V)^{\star}$ could be larger than this, so values of 1.1 and 2.0 are also included. For the $E(B-V)_{\text {old }}^{\star}$ reduction factor, it has been shown that a value of 0.58 is needed for the local universe (Puglisi et al. 2016) instead of the Calzetti et al. (2000) value of 0.44 . A compromise of 0.5 was therefore used.

For the dust emission, the polycyclic aromatic hydrocarbon (PAH) fraction had an increase in range around the default 2.5, so more fractions could be sampled while keeping the number of models reasonable. The minimum scaling factor of the radiation field was similarly given a range to sample with an increase in the smallest value from 1.0 to 5.0. The illuminated fraction was reduced to 0.02, following Ciesla et al. (2015).

The parameters in the AGN model were matched to those used by Ciesla et al. (2015), who undertook a detailed study of AGN host galaxy emission using CIGALE. The number of choices of frac $\mathrm{AGN}_{\mathrm{AGN}}$ was reduced from 14 to 9 , while still covering the same range, to reduce the number of models created by CIGALE to decrease runtime.

A list of parameters, where they differ from default, can be found in Table B.1. 
Table B.1. Parameters used for the various properties in the CIGALE model SEDs where they are different from the default values.

\begin{tabular}{|c|c|c|}
\hline Parameter & Value & Description \\
\hline \multicolumn{3}{|c|}{ Star formation history } \\
\hline$\tau_{\text {main }}$ & $1.0,3.0,5.0$ & e-folding time (main) \\
\hline$\tau_{\text {burst }}$ & $9.0,13.0$ & e-folding time (burst) \\
\hline$f_{\text {burst }}$ & $0.001,0.01,0.1,0.2$ & Burst mass fraction \\
\hline Age & $\begin{array}{l}1.000,1.329,1.768,2.351,3.126 \\
4.157,5.528,7.352,9.776,13.000\end{array}$ & Population age (main) \\
\hline Burst age & $0.001,0.010,0.030,0.100,0.300$ & Population age (burst) \\
\hline \multicolumn{3}{|c|}{ Stellar emission } \\
\hline$Z$ & 0.02 & Metallicity ( 0.02 is solar) \\
\hline \multicolumn{3}{|c|}{ Dust attenuation } \\
\hline$E(B-V)_{\text {young }}^{*}$ & $0.1,0.18,0.33,0.6,1.1,2.0$ & $E(B-V)^{*}$ for young population \\
\hline$E(B-V)_{\text {old }}^{*}$ & 0.5 & Reduction factor in $E(B-V)^{*}$ for old population \\
\hline \multicolumn{3}{|c|}{ Dust emission } \\
\hline$q_{\mathrm{PAH}}$ & $1.12,2.50,3.19$ & Mass fraction of PAH \\
\hline$U_{\min }$ & $5.0,10.0,25.0$ & Minimum scaling factor of the radiation field intensity \\
\hline$\gamma$ & 0.02 & Illuminated fraction \\
\hline \multicolumn{3}{|c|}{ AGN emission } \\
\hline$\tau$ & $1.0,6.0$ & Optical depth at $9.7 \mu \mathrm{m}$ \\
\hline$\gamma$ & 0.0 & $\gamma$ coefficient for the gas density function of the torus ${ }^{a}$ \\
\hline & $0.001^{\circ}, 89.990^{\circ}$ & Angle between equatorial axis and line of sight \\
\hline frac $_{\mathrm{AGN}}$ & $\begin{array}{c}0.0,0.05,0.1,0.2,0.3,0.4,0.5 \\
0.6,0.7\end{array}$ & AGN fraction \\
\hline
\end{tabular}

Notes. All ages and times are in Gyr. ${ }^{(a)}$ Density function of the torus can be found in Fritz et al. (2006) as Eq. (3). 